\title{
Discretizing LTI Descriptor (Regular) Differential Input Systems with Consistent Initial Conditions
}

\author{
Athanasios D. Karageorgos, ${ }^{1}$ Athanasios A. Pantelous, ${ }^{2}$ \\ and Grigoris I. Kalogeropoulos ${ }^{1}$ \\ ${ }^{1}$ Department of Mathematics, University of Athens, 15784 Athens, Greece \\ ${ }^{2}$ Department of Mathematical Sciences, University of Liverpool, Liverpool L69 3BX, UK \\ Correspondence should be addressed to Athanasios A. Pantelous, a.pantelous@liverpool.ac.uk
}

Received 4 April 2009; Accepted 18 January 2010

Academic Editor: Graeme Wake

Copyright (C) 2010 Athanasios D. Karageorgos et al. This is an open access article distributed under the Creative Commons Attribution License, which permits unrestricted use, distribution, and reproduction in any medium, provided the original work is properly cited.

\begin{abstract}
A technique for discretizing efficiently the solution of a Linear descriptor (regular) differential input system with consistent initial conditions, and Time-Invariant coefficients (LTI) is introduced and fully discussed. Additionally, an upper bound for the error $\left\|\underline{x}(k T)-\underline{x}_{k}\right\|$ that derives from the procedure of discretization is also provided. Practically speaking, we are interested in such kind of systems, since they are inherent in many physical, economical and engineering phenomena.
\end{abstract}

\section{Introduction: Preliminary Results}

During the discretization (or sampling) process, we should replace the original continuoustime systems with finite sequences of values at specified discrete-time points. This important process is commonly used whenever the differential systems involve digital inputs, and by having numerical data, the sampling operation and the quantization are necessary. Additionally, the discretization (or sampling) process is occurred whenever significant measurements for the system are obtained in an intermittent fashion. For instance, we can consider a radar tracking system, where there is information about the azimuth and the elevation, which is obtained as the antenna of the radar rotates. Consequently, the scanning operation of the radar produces many important sampled data.

In our approach, we consider the LTI descriptor (or generalized) differential input systems of type

$$
E \underline{\dot{x}}(t)=A \underline{x}(t)+B \underline{u}(t),
$$


where matrices $E, A \in \mathcal{M}(n \times n, \mathbb{F})$ (i.e., $\mathcal{M}$ is the algebra of square matrices with elements in the field $\mathbb{F})$ and $B \in \mathcal{M}(n \times 1, \mathbb{F})$ are constants; the state $\underline{x}(t) \in \mathcal{M}(n \times 1, \mathbb{F})$ has consistent initial conditions. We shall call $\underline{x}_{0}$ a consistent initial condition for (1.1) at $t_{0}$ if there is a solution for (1.1), which is defined on some interval $\left[t_{o}, t_{0}+\gamma\right], \gamma>0$ such that $\underline{x}\left(t_{o}\right)=\underline{x}_{o} \in \mathcal{M}(n \times 1, \mathbb{F})$, the input $\underline{u}(t) \in \mathcal{M}(l \times 1, \mathbb{F})$, and the $\operatorname{det} E=0$ are related to the matrix pencil theory, since its algebraic, geometric, and dynamic properties stem from the structure of the associated pencil, that is, $s E-A$. Moreover, for the sake of simplicity, we set in the sequel $\mathcal{M}_{n}=\mathcal{M}(n \times n ; \mathbb{F})$ and $\mathcal{M}_{n m}=\mathcal{M}(n \times m ; \mathbb{F})$.

Now, in what it follows, the pencil $s E-A$ is regular, that is, $\operatorname{det}(s E-A) \neq 0$.

Practically speaking, descriptor (or generalized) regular (or singular) differential systems constitute a more general class than linear state space systems do. Considering applications, these kinds of systems appear in the modelling procedure of many physical, engineering, mechanical, actuarial, and financial problems. For instance, in engineering, in electrical networks, and in constrained mechanics, the reader may consult [1-6], and so forth. In Economics, the famous Leontief input-output singular dynamic model is well known; see for instance some of the numerous references [2,3,7-12], and so forth.

In this paper, we provide two main research directions that are being summarized briefly below.

(i) First, we want to provide a computationally efficient method for discretizing LTI descriptor regular differential systems with input signals and consistent initial conditions. The consistency of the initial conditions is necessary, because it eliminates completely the possibility to appear as a distributional expression for the solution of system (1.1), that is, Dirac delta functions and its derivatives.

(ii) Second, according to the authors' knowledge, for the first time an upper bound for the error $\left\|\underline{x}(k T)-\underline{x}_{k}\right\|$, which is derived during the discretization process, is finally obtained. Consequently, an analytic expression that penalizes our choice for the sampling period $T$ is provided through the notion of the $\|\cdot\|$-norm of the difference between the continuous-time solution $\underline{x}(k T)$, at time $t=k T$, and the relevant discrete-time points $\underline{x}_{k}$.

This investigation is relevant to and it extends further the recent work proposed by Karampetakis; see [13] and Kalogeropoulos et al.; see [14]. Concerning the mathematical tools, only the Weierstrass canonical form (WCF) and some fundamental elements of matrix pencil theory are required.

Recently, in $[15,16]$, several numerical issues of the WCF of a regular matrix pencil are presented and discussed. Thus, briefly speaking, in these research papers, two important computational tools are considered: (a) the QZ algorithm to specify the required root range of the pencil and (b) the updating technique to compute the index of annihilation; see [16]. The proposed updating technique takes advantages of the already computed rank of the sequences of matrices that appears during our procedure reducing significantly the required floating-point operations. The algorithms are implemented in a numerical stable manner, giving efficient results.

For reasons of convenience, some basic concepts and definitions from matrix pencil theory are introduced; see for more details [2-4, 17-22] et al. Thus, the class of strict equivalence is characterized by a uniquely defined element, known as a WCF, that is, $s E_{w}-A_{w}$. Consequently, when the pencil $s E-A$ is regular, we have elementary divisors of the following type: 
(i) e.d. of the type $s^{d}, d \in \mathbb{N}$, are called zero finite elementary divisors (z. f.e.d.);

(ii) e.d. of the type $(s-a)^{c}, a \neq 0, c \in \mathbb{N}$ are called nonzero finite elementary divisors (nz. f.e.d.);

(iii) e.d. of the type $\widehat{s}^{q}$ are called infinite elementary divisors (i.e.d.).

Then, the Weierstrass canonical form of the regular pencil $s E-A$ is defined by

$$
s E_{w}-A_{w} \triangleq \operatorname{block} \operatorname{diag}\left\{s I_{p}-J_{p}, s H_{q}-I_{q}\right\}
$$

where the first normal Jordan type block $s I_{p}-J_{p}$ is uniquely defined by the set of f.e.d.

$$
\left(s-a_{1}\right)^{p_{1}}, \ldots,\left(s-a_{v}\right)^{p_{v}}, \quad \sum_{j=1}^{v} p_{j}=p
$$

of $s F-G$ and has the form

$$
s I_{p}-J_{p} \triangleq \text { block diag }\left\{s I_{p_{1}}-J_{p_{1}}\left(a_{1}\right), \ldots, s I_{p_{v}}-J_{p_{v}}\left(a_{v}\right)\right\}
$$

And the $q$ blocks of the second uniquely defined block $s H_{q}-I_{q}$ correspond to the i.e.d.

$$
(\widehat{s})^{q_{1}}, \ldots,(\widehat{s})^{q_{\sigma}}, \quad \sum_{j=1}^{\sigma} q_{j}=q
$$

of $s E-A$ and has the form

$$
s H_{q}-I_{q} \triangleq \text { block diag }\left\{s H_{q_{1}}-I_{q_{1}}, \ldots, s H_{q_{\sigma}}-I_{q_{\sigma}}\right\} \text {. }
$$

Thus the $H_{q}$ is a nilpotent matrix of index $q^{*}=\max \left\{q_{j}: j=1,2, \ldots, \sigma\right\}$, where $H_{q}^{q^{*}}=\mathbb{O}$ and $I_{p_{i}}, J_{p_{i}}\left(a_{i}\right), H_{q_{i}}$ are the matrices

$$
\begin{gathered}
I_{p_{i}}=\left[\begin{array}{cccc}
1 & 0 & \cdots & 0 \\
0 & 1 & \cdots & 0 \\
\vdots & \vdots & \ddots & \vdots \\
0 & 0 & \cdots & 1
\end{array}\right] \in \mathcal{M}\left(p_{i} \times p_{i}, \mathbb{R}\right), \quad H_{q_{i}}=\left[\begin{array}{ccccc}
0 & 1 & 0 & \cdots & 0 \\
0 & 0 & 1 & \cdots & 0 \\
\vdots & \vdots & \ddots & \vdots & \vdots \\
0 & 0 & 0 & 0 & 1 \\
0 & 0 & 0 & 0 & 0
\end{array}\right] \in \mathcal{M}\left(q_{i} \times q_{i}, \mathbb{R}\right), \\
J_{p_{i}}\left(a_{i}\right)=\left[\begin{array}{ccccc}
a_{i} & 1 & 0 & \cdots & 0 \\
0 & a_{i} & 1 & \cdots & 0 \\
\vdots & \vdots & \ddots & \vdots & \vdots \\
0 & 0 & 0 & a_{i} & 1 \\
0 & 0 & 0 & 0 & a_{i}
\end{array}\right] \in \mathcal{M}\left(p_{i} \times p_{i}, \mathbb{C}\right) .
\end{gathered}
$$


Now, considering $[4,17,18]$, and the transformation

$$
\underline{x}(t)=Q \underline{y}(t)
$$

we obtain the following result. (Note that matrix $Q$ is not unique. However, different matrices $Q$ are mutually related.) We use the above transformation, (1.8), since we want to create the WCF; see [17].

Theorem 1.1 (see [4, 23]). System (1.1) has the following solution:

$$
\underline{x}(t)=Q_{n, p}\left\{e^{J_{p}\left(t-t_{o}\right)} \underline{y}_{p}\left(t_{o}\right)+\int_{t_{o}}^{t} e^{J_{p}(t-s)} B_{p, l} \underline{u}(s) d s\right\}-Q_{n, q} \sum_{i=0}^{q^{*}-1} H_{q}^{i} B_{q, l} \underline{u}^{(i)}(t),
$$

where $Q=\left[\begin{array}{ll}Q_{n, p} & Q_{n, q}\end{array}\right], B=\left[\begin{array}{l}B_{p, l} \\ B_{q, l}\end{array}\right]$, and $\underline{y}\left(t_{o}\right)=\left[\begin{array}{l}\underline{y}_{p}\left(t_{o}\right) \\ \underline{y}_{q}\left(t_{o}\right)\end{array}\right]=Q^{-1} \underline{x}\left(t_{o}\right)$. we obtain

However, (1.9) should be transposed to (1.12), which is practically more useful. Thus,

$$
\begin{aligned}
\underline{x}(t)= & Q_{n, p} e^{J_{p}\left(t-t_{o}\right)} \underline{y}_{p}\left(t_{o}\right)+Q_{n, q} \underline{y}_{q}\left(t_{o}\right)+Q_{n, p} \int_{t_{o}}^{t} e^{J_{p}(t-s)} B_{p, l} \underline{u}(s) d s \\
& -Q_{n, q} \underline{y}_{q}\left(t_{o}\right)-Q_{n, q} \sum_{i=0}^{q^{*}-1} H_{q}^{i} B_{q, l} \underline{u}^{(i)}(t) \\
= & {\left[\begin{array}{ll}
Q_{n, p} & Q_{n, q}
\end{array}\right]\left[\begin{array}{cc}
e^{J_{p}\left(t-t_{o}\right)} & \mathbb{O}_{p, q} \\
\mathbb{O}_{q, p} & I_{q}
\end{array}\right]\left[\begin{array}{l}
\underline{y}_{p}^{\left(t_{o}\right)} \\
\underline{y}_{q}\left(t_{o}\right)
\end{array}\right]+Q_{n, p} \int_{t_{o}}^{t} e^{J_{p}(t-s)} B_{p, l} \underline{u}(s) d s } \\
& +Q_{n, q}\left(\underline{-}_{q}\left(t_{o}\right)-\sum_{i=0}^{q^{*}-1} H_{q}^{i} B_{q, l} \underline{u}^{(i)}(t)\right) .
\end{aligned}
$$

In order the system (1.1) to obtain consistent initial conditions, we should consider that

$$
\left[\begin{array}{l}
\underline{y}_{p}\left(t_{o}\right) \\
\underline{y}_{q}\left(t_{o}\right)
\end{array}\right]=Q^{-1} \underline{x}\left(t_{o}\right), \quad \underline{y}_{q}\left(t_{o}\right)=\sum_{i=0}^{q^{*}-1} H_{q}^{i} B_{q,} \underline{u}^{(i)}\left(t_{o}\right)
$$

Consequently, we obtain the desired expression for (1.9), that is,

$$
\begin{aligned}
\underline{x}(t)= & Q\left[\begin{array}{cc}
e^{J_{p}\left(t-t_{o}\right)} & \mathbb{O}_{p, q} \\
\mathbb{O}_{q, p} & I_{q}
\end{array}\right] Q^{-1} \underline{x}\left(t_{o}\right)+Q_{n, p} \int_{t_{o}}^{t} e^{J_{p}(t-s)} B_{p, l} \underline{u}(s) d s \\
& +Q_{n, q} \sum_{i=0}^{q^{*}-1} H_{q}^{i} B_{q, l}\left(\underline{u}^{(i)}\left(t_{o}\right)-\underline{u}^{(i)}(t)\right) .
\end{aligned}
$$


Moreover, by definition, the state-transition matrix of the autonomous linear descriptor differential system, that is, $E \underline{\dot{x}}(t)=A \underline{x}(t)$, is given by

$$
\Phi\left(t, t_{o}\right)=Q\left[\begin{array}{cc}
e^{J_{p}\left(t-t_{o}\right)} & \mathbb{O}_{p, q} \\
\mathbb{O}_{q, p} & I_{q}
\end{array}\right] Q^{-1}
$$

Finally, after some simple algebraic calculations, we obtain another more elegance form for the solution of system (1.1), that is,

$$
\underline{x}(t)=\Phi\left(t, t_{o}\right) \underline{x}\left(t_{o}\right)+\int_{t_{o}}^{t} \Phi(t, s) Q_{n, p} B_{p, l} \underline{u}(s) d s+Q_{n, q} \sum_{i=0}^{q^{*}-1} H_{q}^{i} B_{q, l}\left(\underline{u}^{(i)}\left(t_{o}\right)-\underline{u}^{(i)}(t)\right),
$$

where $\underline{x}\left(t_{o}\right) \in \tau$ belongs to the space of consistent initial conditions of system (1.1), that is,

$$
\tau=\left\{\underline{x} \in \mathcal{M}(n \times 1, \mathbb{C}): \underline{x}=Q_{n, p} \underline{C}_{p}-Q_{n, q} \sum_{i=0}^{q^{*}-1} H_{q}^{i} B_{q,} \underline{u}^{(i)}\left(t_{o}\right), \text { with } \underline{C}_{p} \in \mathcal{M}(p \times 1, \mathbb{C})\right\}
$$

\section{Discretisation of Nonhomogeneous LTI Descriptor Regular Differential System}

In this section, we provide a computationally efficient method for the analytic discretization of LTI descriptor regular differential systems with input signal and consistent initial conditions. In the vast literature of descriptor systems, Karampetakis has proposed two discretization methods for regular systems without (see [13, Section 3]) and with inputs (see [13, Section 4]), which are based on the research work by Koumboulis and Mertzios, see [24], concerning the solution of regular systems in terms of the Laurent expansion terms of $(s E-A)^{-1}$. The methodology of both papers, that is, [13] and the present, is equivalent, since they use the zero-order hold approximation.

Moreover, specifically for regular systems, Rachid, see [21], has proposed a different method, which discretizes the exact solution using Euler approximation techniques.

However, in the present paper, a different to the above research works discretization technique for LTI descriptor differential input systems is presented, which extends further the research work $[8,14]$.

First, we denote $T>0$ as the constant sampling period. Without loss of generality and because of the complex notations that follow, we assume that $t_{o}=0$, and the input function $\underline{u}(t)$ changes only through the time moments $t_{k}=k T$ for $k=1,2, \ldots$, that is,

$$
\underline{u}(t)=\underline{u}(k T)=\underline{u}_{k}
$$

for every $t \in[k T,(k+1) T)$.

In more details, (2.1) implies that the input $\underline{u}(t)$ is being inserted into a mechanism of zero-order change after the end of each sampling process. Thus, the input remains constant from time $t_{k}=k T$ until the next sampling period at time $t_{k+1}=(k+1) T$ takes place. 
Thus, the solution (1.14) is given by

$$
\underline{x}(t)=\Phi(t, 0) \underline{x}_{0}+\int_{0}^{t} \Phi(t, s) Q_{n, p} B_{p, l} \underline{u}(s) d s+Q_{n, q} \sum_{i=0}^{q^{*}-1} H_{q}^{i} B_{q, l}\left(\underline{u}^{(i)}(0)-\underline{u}^{(i)}(t)\right)
$$

where $\underline{x}_{0}=\underline{x}(0) \in \tau$.

Moreover, hereafter, we use the notation

$$
\underline{x}_{k} \equiv \underline{x}(k T) \quad \forall k=0,1,2, \ldots
$$

The following theorem provides us with an analytic formula based on (2.2).

Theorem 2.1. An analytic formula for the discretized solution (2.2) of system (1.1) is given by (2.4)

$$
\begin{aligned}
\underline{x}_{k}= & \Phi(k T, 0) \underline{x}_{0}+\sum_{j=0}^{k-1} \int_{0}^{T} \Phi(j T+\lambda, 0) d \lambda \cdot Q_{n, p} B_{p, l} \underline{u}_{k-1-j} \\
& +Q_{n, q} \sum_{i=0}^{q^{*}-1} H_{q}^{i} B_{q, l}\left(\underline{u}^{(i)}(0)-\underline{u}^{(i)}(k T)\right) .
\end{aligned}
$$

Proof. First, we consider (2.2) at time moments $t=k T$ and $t=(k+1) T$.

Thus, we obtain for the time $t=k T$

$$
\underline{x}_{k}=\Phi(k T, 0) \underline{x}_{0}+\int_{0}^{k T} \Phi(k T, s) Q_{n, p} B_{p, l} \underline{u}(s) d s+Q_{n, q} \sum_{i=0}^{q^{*}-1} H_{q}^{i} B_{q, l}\left(\underline{u}^{(i)}(0)-\underline{u}^{(i)}(k T)\right)
$$

and for the time $t=(k+1) T$

$$
\begin{aligned}
\underline{x}_{k+1}= & \Phi((k+1) T, 0) \underline{x}_{0}+\int_{0}^{(k+1) T} \Phi((k+1) T, s) Q_{n, p} B_{p, l} \underline{u}(s) d s \\
& +Q_{n, q} \sum_{i=0}^{q^{*}-1} H_{q}^{i} B_{q, l}\left(\underline{u}^{(i)}(0)-\underline{u}^{(i)}((k+1) T)\right) .
\end{aligned}
$$

The state-transition matrix (1.13) satisfies the following two properties:

$$
\begin{aligned}
& \Phi(T, 0) \cdot \Phi(k T, 0)=\Phi((k+1) T, 0) \\
& \Phi(T, 0) \cdot \Phi(k T, s)=\Phi((k+1) T, s)
\end{aligned}
$$


Now, we multiply (2.5) from the left with $\Phi(T, 0)$, and we consider (2.7) and (2.8). Thus, we obtain

$$
\begin{aligned}
\Phi(T, 0) \underline{x}_{k}= & \Phi((k+1) T, 0) \underline{x}_{0}+\int_{0}^{k T} \Phi((k+1) T, s) Q_{n, p} B_{p, l} \underline{u}(s) d s \\
& +\Phi(T, 0) Q_{n, q} \sum_{i=0}^{q^{*}-1} H_{q}^{i} B_{q, l}\left(\underline{u}^{(i)}(0)-\underline{u}^{(i)}(k T)\right) .
\end{aligned}
$$

Consequently, by also using (2.6) and (2.9), we take the difference

$$
\begin{aligned}
\underline{x}_{k+1}-\Phi(T, 0) \underline{x}_{k}= & \int_{k T}^{(k+1) T} \Phi((k+1) T, s) Q_{n, p} B_{p, l} \underline{u}(s) d s \\
& +Q_{n, q} \sum_{i=0}^{q^{*}-1} H_{q}^{i} B_{q, l}\left(u^{(i)}(0)-u^{(i)}((k+1) T)\right) \\
& -\Phi(T, 0) Q_{n, q} \sum_{i=0}^{q^{*}-1} H_{q}^{i} B_{q, l}\left(\underline{u}^{(i)}(0)-\underline{u}^{(i)}(k T)\right) .
\end{aligned}
$$

Afterwards, we should define

$$
\begin{gathered}
Q^{-1}=\left[\begin{array}{c}
\widetilde{Q}_{p, n} \\
\widetilde{Q}_{p, n}
\end{array}\right], \\
Q^{-1} Q=\left[\begin{array}{c}
\widetilde{Q}_{p, n} \\
\widetilde{Q}_{q, n}
\end{array}\right] Q=\left[\begin{array}{c}
\widetilde{Q}_{p, n} \\
\widetilde{Q}_{q, n}
\end{array}\right]\left[\begin{array}{ll}
Q_{n, p} & Q_{n, q}
\end{array}\right]=\left[\begin{array}{cc}
\widetilde{Q}_{p, n} Q_{n, p} & \widetilde{Q}_{p, n} Q_{n, q} \\
\widetilde{Q}_{q, n} Q_{n, p} & \widetilde{Q}_{q, n} Q_{n, q}
\end{array}\right]=I_{n}
\end{gathered}
$$

with

$$
\begin{array}{ll}
\widetilde{Q}_{p, n} Q_{n, p}=I_{p}, & \widetilde{Q}_{p, n} Q_{n, q}=\mathbb{O}_{p, q}, \\
\widetilde{Q}_{q, n} Q_{n, p}=\mathbb{O}_{q, p}, & \widetilde{Q}_{q, n} Q_{n, q}=I_{q} .
\end{array}
$$

Thus,

$$
\begin{aligned}
\Phi(T, 0) Q_{n, q} & =Q\left[\begin{array}{cc}
e^{J_{p} T} & \mathbb{O}_{p, q} \\
\mathbb{O}_{q, p} & I_{q}
\end{array}\right] Q^{-1} Q_{n, q}=\left[\begin{array}{ll}
Q_{n, p} & Q_{n, q}
\end{array}\right]\left[\begin{array}{cc}
e^{J_{p} T} & \mathbb{O}_{p, q} \\
\mathbb{O}_{q, p} & I_{q}
\end{array}\right]\left[\begin{array}{cc}
\tilde{Q}_{p n} & Q_{n q} \\
\widetilde{Q}_{q n} & Q_{n q}
\end{array}\right] \\
& =\left[\begin{array}{ll}
Q_{n, p} & Q_{n, q}
\end{array}\right]\left[\begin{array}{cc}
e^{J_{p} T} & \mathbb{O}_{p, q} \\
\mathbb{O}_{q, p} & I_{q}
\end{array}\right]\left[\begin{array}{c}
\mathbb{O}_{p, q} \\
I_{q}
\end{array}\right]=\left[Q_{n, p}, Q_{n, q}\right]\left[\begin{array}{c}
\mathbb{O}_{p, q} \\
I_{q}
\end{array}\right]=Q_{n, q} .
\end{aligned}
$$


So $\Phi(T, 0) Q_{n, q}=Q_{n, q}$. Consequently, (2.10) is transformed to (2.14)

$$
\begin{aligned}
\underline{x}_{k+1}= & \Phi(T, 0) \underline{x}_{k}+\int_{k T}^{(k+1) T} \Phi((k+1) T, s) Q_{n, p} B_{p, l} \underline{u}(s) d s \\
& +Q_{n, q} \sum_{i=0}^{q^{*}-1} H_{q}^{i} B_{q, l}\left(\underline{u}^{(i)}(k T)-\underline{u}^{(i)}((k+1) T)\right) .
\end{aligned}
$$

Note that by denoting $s=k T+w$, the integral $\int_{k T}^{(k+1) T} \Phi((k+1) T, s) Q_{n, p} B_{p, l} \underline{u}(s) d s$ is given by

$$
\begin{aligned}
& \int_{k T}^{(k+1) T} \Phi((k+1) T, s) Q_{n, p} B_{p, l} \underline{u}(s) d s=\int_{0}^{T} \Phi((k+1) T, k T+w) Q_{n, p} B_{p, l} \underline{u}(k T+w) d w \\
& \stackrel{(2.1)}{=} \int_{0}^{T} \Phi((k+1) T, k T+w) d w \cdot Q_{n, p} B_{p, l} \underline{u}_{k} .
\end{aligned}
$$

Moreover, by using (2.7) and (2.8), we obtain

$$
\int_{0}^{T} \Phi((k+1) T, k T+w) d w=\int_{0}^{T} \Phi((k+1) T, k T) \Phi(0, w) d w
$$

And considering again (2.8), we have

$$
\Phi((k+1) T, k T)=\Phi(T, 0) \cdot \Phi(k T, k T)=\Phi(T, 0)
$$

because $\Phi(k T, k T)=I_{n}$. Finally, we obtain

$$
\begin{aligned}
\int_{0}^{T} \Phi((k+1) T, k T+w) d w & =\int_{0}^{T} \Phi(T, 0) \Phi(0, w) d w=\int_{0}^{T} \Phi(T, w) d w \\
& =\int_{0}^{T} \Phi(T-w, 0) d w=\int_{0}^{T} \Phi(\lambda, 0) d \lambda
\end{aligned}
$$

when we define $\lambda=T-w$. Thus,

$$
\int_{k T}^{(k+1) T} \Phi((k+1) T, s) Q_{n, p} B_{p, l} \underline{u}(s) d s=\int_{0}^{T} \Phi(\lambda, 0) d \lambda \cdot Q_{n, p} B_{p, l} \underline{u}_{k} .
$$

Combining (2.14) and (2.19), we take the recursive formula (2.20)

$$
\begin{aligned}
\underline{x}_{k+1}= & \Phi(T, 0) \underline{x}_{k}+\int_{0}^{T} \Phi(\lambda, 0) d \lambda \cdot Q_{n, p} B_{p, l} \underline{u}_{k} \\
& +Q_{n, q} \sum_{i=0}^{q^{*}-1} H_{q}^{i} B_{q, l}\left(\underline{u}^{(i)}(k T)-\underline{u}^{(i)}((k+1) T)\right) .
\end{aligned}
$$


Then, the formula (2.4) is obtained by (2.20). Analytically,

(i) for $k=0$, we take

$\underline{x}_{1}=\Phi(T, 0) \underline{x}_{0}+\int_{0}^{T} \Phi(\lambda, 0) d \lambda \cdot Q_{n, p} B_{p, l} \underline{u}_{0}+Q_{n, q} \sum_{i=0}^{q^{*}-1} H_{q}^{i} B_{q, l}\left(\underline{u}^{(i)}(0)-\underline{u}^{(i)}(T)\right) ;$

(ii) for $k=1$, we have

$$
\begin{aligned}
\underline{x}_{2}= & \Phi(T, 0) \underline{x}_{1}+\int_{0}^{T} \Phi(\lambda, 0) d \lambda \cdot Q_{n, p} B_{p, l} \underline{u}_{1}+Q_{n, q} \sum_{i=0}^{q^{*}-1} H_{q}^{i} B_{q, l}\left(\underline{u}^{(i)}(T)-\underline{u}^{(i)}(2 T)\right) \\
= & \Phi(T, 0) \Phi(T, 0) \underline{x}_{0}+\int_{0}^{T} \Phi(T, 0) \Phi(\lambda, 0) d \lambda \cdot Q_{n, p} B_{p, l} \underline{u}_{0} \\
& +\Phi(T, 0) Q_{n, q} \sum_{i=0}^{q^{*}-1} H_{q}^{i} B_{q, l}\left(\underline{u}^{(i)}(0)-\underline{u}^{(i)}(T)\right) \\
& +\int_{0}^{T} \Phi(\lambda, 0) d \lambda \cdot Q_{n, p} B_{p, l} \underline{u}_{1}+Q_{n, q} \sum_{i=0}^{q^{*}-1} H_{q}^{i} B_{q, l}\left(\underline{u}^{(i)}(T)-\underline{u}^{(i)}(2 T)\right) .
\end{aligned}
$$

Since $\Phi(T, 0) Q_{n, q}=Q_{n, q}$, we conclude to

$$
\begin{aligned}
\underline{x}_{2}= & \Phi(2 T, 0) \underline{x}_{0}+\int_{0}^{T} \Phi(T+\lambda, 0) d \lambda \cdot Q_{n, p} B_{p, l} \underline{u}_{0} \\
& +\int_{0}^{T} \Phi(\lambda, 0) d \lambda \cdot Q_{n p} B_{p, l} \underline{u}_{1}+Q_{n, q} \sum_{i=0}^{q^{*}-1} H_{q}^{i} B_{q, l}\left(\underline{u}^{(i)}(0)-\underline{u}^{(i)}(2 T)\right) ;
\end{aligned}
$$

(iii) for $k=3$, we take

$$
\begin{aligned}
\underline{x}_{3}= & \Phi(3 T, 0) \underline{x}_{0}+\int_{0}^{T} \Phi(2 T+\lambda, 0) d \lambda \cdot Q_{n, p} B_{p, l} \underline{u}_{0}+\int_{0}^{T} \Phi(T+\lambda, 0) d \lambda \cdot Q_{n, p} \cdot B_{p, l} \underline{u}_{1} \\
& +\int_{0}^{T} \Phi(\lambda, 0) d \lambda \cdot Q_{n, p} B_{p, l} \underline{u}_{2}+Q_{n, q} \sum_{i=0}^{q^{*}-1} H_{q}^{i} B_{q, l}\left(\underline{u}^{(i)}(0)-\underline{u}^{(i)}(3 T)\right) .
\end{aligned}
$$

Continuing as above, we finally obtain the analytic formula (2.4).

Now, in order to complete the discretization process of the solution (2.2) of the system (1.1), we should replace the $i$ th-order derivative $\underline{u}_{k}^{(i)} \equiv \underline{u}^{(i)}(k T)$ with expressions of $\underline{u}(k T)$. Thus, the following result is used, see [25],

$$
\underline{u}_{k}^{(i)}=\underline{u}^{(i)}(k T) \approx \frac{\sum_{j=0}^{i}(-1)^{j}\left(\begin{array}{l}
i \\
j
\end{array}\right) \underline{u}_{k-i+j}}{T^{i}} .
$$


Profoundly, based on (2.25), the following difference can be easily proved.

$$
\underline{u}_{k}^{(i)}-\underline{u}_{k+1}^{(i)} \approx \frac{\sum_{j=0}^{i+1}(-1)^{j+1}\left(\begin{array}{c}
i+1 \\
j
\end{array}\right) \underline{u}_{k-i+j}}{T^{i+1}} .
$$

Remark 2.2. The discrete-time solution which represents (2.2) of the continuous-time system (1.1) is given by (2.27), (see also [13, Theorem 3])

$$
\begin{aligned}
\underline{x}_{k}= & \Phi(k T, 0) \underline{x}_{0}+\sum_{j=0}^{k-1} \int_{0}^{T} \Phi(j T+\lambda, 0) d \lambda \cdot Q_{n, p} B_{p, l} \underline{u}_{k-1-j} \\
& +Q_{n, q} \sum_{i=0}^{q^{*}-1} \frac{1}{T^{i+1}} H_{q}^{i} B_{q, l} \sum_{j=0}^{i+1}(-1)^{j+1}\left(\begin{array}{c}
i+1 \\
j
\end{array}\right) \underline{u}_{k-1+j} .
\end{aligned}
$$

Remark 2.3. Equation (2.20) reminds us with a new discrete-time system of the form

$$
\underline{x}_{k+1}=A \underline{x}_{k}+B(\sigma) \underline{u}_{k^{\prime}}
$$

where $A=\Phi(T, 0)$, whereas the differences $\underline{u}^{(i)}(k T)-\underline{u}^{(i)}((k+1) T)$ can be replaced by $(2.26)$ in order to get the matrix $B(\sigma)$. This approach is equivalent to Theorem 3.7 in [13].

Remark 2.4. The induction method (show that it is true for $n=0$; assume that it is true for $n=k$; try to prove that it is true for $n=k+1$ ) can prove (2.4). However, we strongly believe that our approach seems to be more natural, since (2.4) is analytically constructed.

In the next section, according to the authors' knowledge, an upper bound for the error $\left\|\underline{x}(k T)-\underline{x}_{k}\right\|$, which is derived during the discretization process, is provided for the first time.

\section{Error Analysis for the Discretization (or Sampling) Process}

In this section, we provide an analytic expression that penalizes our choice for the sampling period $T$ through the notion of the Euclidean $\|\cdot\|$-norm of the difference between the continuous-time solution (1.13), at time $t=k T, \underline{x}(k T)$ and the relevant discrete-time points $\underline{x}_{k}$, see (2.4), that is, we are interested in the difference $\left\|\underline{x}(k T)-\underline{x}_{k}\right\|$.

Following the results of the previous sections, we obtain (3.2). Analytically, we firstly note that the comparison of the solutions is provided at time moment $t=k T$.

The solution (1.13) at time moment $t=k T$ for $k=1,2,3, \ldots$, is given by (3.1)

$$
\begin{aligned}
\underline{x}(k T)= & \Phi(k T, 0) \underline{x}_{0}+\int_{0}^{k T} \Phi(k T, s) Q_{n, p} B_{p, l} \underline{u}(s) d s \\
& +Q_{n, q} \sum_{i=0}^{q^{*}-1} H_{q}^{i} B_{q, l}\left(\underline{u}^{(i)}(0)-\underline{u}^{(i)}(k T)\right)
\end{aligned}
$$


Combining (2.4) and (3.1), we obtain the following important expression:

$$
\begin{aligned}
\underline{x}(k T)-\underline{x}_{k}= & \int_{0}^{k T} \Phi(k T, s) Q_{n, p} B_{p, l} \underline{u}(s) d s \\
& -\sum_{j=0}^{k-1} \int_{0}^{T} \Phi(j T+\lambda, 0) d \lambda \cdot Q_{n, p} B_{p, l} \underline{u}_{k-1-j}
\end{aligned}
$$

(note that it is not important to consider the completely discretized equation (2.27) instead of (2.4), since the second part with the ith-order derivatives is eliminated).

In order to compare the two solutions at a fixed time moments, the following lemmas are stated and straightforwardly proved.

Lemma 3.1. The equality (3.3) holds

$$
\int_{0}^{k T} \Phi(k T, s) Q_{n, p} B_{p, l} \underline{u}(s) d s=\sum_{j=0}^{k-1} \int_{j T}^{(j+1) T} \Phi(k T, s) d s \cdot Q_{n, p} B_{p, l} \underline{u} j .
$$

Proof. Consider that

$$
\begin{aligned}
\int_{0}^{k T} \Phi(k T, s) Q_{n, p} B_{p, l} \underline{u}(s) d s= & \int_{0}^{T} \Phi(k T, s) Q_{n, p} B_{p, l} \underline{u}(s) d s+\int_{T}^{2 T} \Phi(k T, s) Q_{n, p} B_{p, l} \underline{u}(s) d s \\
& +\cdots+\int_{(k-1) T}^{k T} \Phi(k T, s) Q_{n, p} B_{p, l} \underline{u}(s) d s .
\end{aligned}
$$

Since (2.1) holds, we take

$$
\begin{aligned}
\int_{0}^{k T} \Phi(k T, s) Q_{n, p} B_{p, l} \underline{u}(s) d s= & \int_{0}^{T} \Phi(k T, s) Q_{n, p} B_{p, l} \underline{u}_{0} d s+\int_{T}^{2 T} \Phi(k T, s) Q_{n, p} B_{p, l} \underline{u}_{1} d s \\
& +\cdots+\int_{(k-1) T}^{k T} \Phi(k T, s) Q_{n, p} B_{p, l} \underline{u}_{k-1} d s \\
= & \int_{0}^{T} \Phi(k T, s) d s \cdot Q_{n, p} B_{p, l} \underline{u}_{0}+\int_{T}^{2 T} \Phi(k T, s) d s \cdot Q_{n, p} B_{p, l} \underline{u}_{1} \\
& +\cdots+\int_{(k-1) T}^{k T} \Phi(k T, s) d s \cdot Q_{n, p} B_{p, l} \underline{u}_{k-1} \cdot
\end{aligned}
$$

Thus, (3.3) derives. 
Lemma 3.2. The equality (3.6) holds

$$
\int_{j T}^{(j+1) T} \Phi(k T, s) d s=\int_{0}^{T} \Phi((k-j) T-\lambda, 0) d \lambda .
$$

Proof. We define that $s=j T+\lambda$; if $s=j T$, then $\lambda=0$; if $s=(j+1) T$, then $\lambda=T$.

Thus, we obtain

$$
\int_{j T}^{(j+1) T} \Phi(k T, s) d s=\int_{0}^{T} \Phi(k T, j T+\lambda) d \lambda=\int_{0}^{T} \Phi((k-j) T-\lambda, 0) d \lambda
$$

since it can easily be proven that

$$
\Phi(k T, j T+\lambda)=\Phi((k-j) T-\lambda, 0) .
$$

Now, we consider Lemmas 3.1 and 3.2, and (3.2) is transformed to (3.9)

$$
\begin{aligned}
\underline{x}(k T)-\underline{x}_{k}= & \sum_{j=0}^{k-1} \int_{0}^{T} \Phi((k-j) T-\lambda, 0) d \lambda \cdot Q_{n, p} B_{p, l} \underline{u}_{j} \\
& -\sum_{j=0}^{k-1} \int_{0}^{T} \Phi(j T+\lambda, 0) d \lambda \cdot Q_{n, p} B_{p, l} \underline{u}_{k-1-j} .
\end{aligned}
$$

Lemma 3.3. The equality (3.10) holds

$$
\sum_{j=0}^{k-1} \int_{0}^{T} \Phi(j T+\lambda, 0) d \lambda \cdot Q_{n, p} B_{p, l} \underline{u}_{k-1-j}=\sum_{j=0}^{k-1} \int_{0}^{T} \Phi((k-1-j) T+\lambda, 0) d \lambda \cdot Q_{n, p} B_{p, l} \underline{u}_{j} .
$$

Proof. Making the transformation $k-1-j=i$, we obtain $j=k-1-i$.

Since $j=0,1, \ldots, k-1$, then $i=k-1, k-2, \ldots, 0$. So, we obtain

$$
\sum_{j=0}^{k-1} \int_{0}^{T} \Phi(j T+\lambda, 0) d \lambda \cdot Q_{n, p} B_{p, l} \underline{u}_{k-1-j}=\sum_{i=0}^{k-1} \Phi((k-1-i) T+\lambda, 0) d \lambda \cdot Q_{n, p} B_{p, l} \underline{u}_{i} .
$$

Now, if we also define that $j=i,(3.10)$ holds. 
Following the results of Lemma 3.3, (3.9) is obtained the following expression:

$$
\underline{x}(k T)-\underline{x}_{k}=\sum_{j=0}^{k-1} \int_{0}^{T}(\Phi((k-j) T-\lambda, 0)-\Phi((k-1-j) T+\lambda, 0)) d \lambda \cdot Q_{n, p} B_{p, l} \underline{u}_{j} .
$$

Moreover, it is true that

$$
\Phi((k-j) T-\lambda, 0)-\Phi((k-j) T-T+\lambda, 0)=\Phi((k-j) T, 0)(\Phi(-\lambda, 0)-\Phi(\lambda-T, 0)) .
$$

Consequently, (3.12) is given by

$$
\underline{x}(k T)-\underline{x}_{k}=\sum_{j=0}^{k-1} \Phi((k-j) T, 0) \cdot \int_{0}^{T}(\Phi(-\lambda, 0)-\Phi(\lambda-T, 0)) d \lambda \cdot Q_{n, p} B_{p, l} \underline{u}_{j}
$$

which is practically more significant in what follows.

For the calculation of the upper bound of the difference $\underline{x}(k T)-\underline{x}_{k}$, we use the Euclidean $\|\cdot\|$-norm for vectors. Thus, we take

$$
\begin{aligned}
\left\|\underline{x}(k T)-\underline{x}_{k}\right\| & =\left\|\sum_{j=0}^{k-1} \Phi((k-j) T, 0) \cdot \int_{0}^{T}(\Phi(-\lambda, 0)-\Phi(\lambda-T, 0)) d \lambda \cdot Q_{n, p} B_{p, l} \underline{u}_{j}\right\| \\
& \leq \sum_{j=0}^{k-1}\|\Phi((k-j) T, 0)\| \cdot \int_{0}^{T}\|\Phi(-\lambda, 0)-\Phi(\lambda-T, 0)\| d \lambda\left\|Q_{n, p}\right\| \cdot\left\|B_{p, l}\right\| \cdot\left\|\underline{u}_{j}\right\| .
\end{aligned}
$$

Now, for the calculation of the upper bound of the difference (3.15), one more lemma and some propositions should be considered.

Lemma 3.4. The inequality (3.16) holds

$$
\left\|e^{J_{p} t}\right\| \leq \sum_{i=1}^{v}\left[e^{\alpha_{i} t}\left(\sum_{m=0}^{p_{i}-1}\left(\left(\frac{t^{m}}{m !}\right)^{2}\left(p_{i}-m\right)\right)\right)^{1 / 2}\right]
$$

Proof. Considering (1.7), the block matrix $J_{p}$ is given by

$$
J_{p}=\left[\begin{array}{llll}
J_{p_{1}}\left(\alpha_{1}\right) & & & \\
& J_{p_{2}}\left(\alpha_{2}\right) & & \\
& & \ddots & \\
& & & J_{p_{v}}\left(\alpha_{v}\right)
\end{array}\right],
$$


where $\alpha_{1}, \alpha_{2}, \ldots, \alpha_{v}$ are nonzero finite elementary divisors of matrix pencil $s E-A$; consult Section 1 for more details. Moreover,

$$
J_{p_{i}}\left(\alpha_{i}\right)=\left[\begin{array}{ccccc}
\alpha_{i} & 1 & & \\
& & & & \\
& \alpha_{i} & \ddots & \\
& & \ddots & \vdots \\
& & & \alpha_{i}
\end{array}\right]_{p_{i} \times p_{i}} \quad \text { for every } i=1,2, \ldots, \mathrm{v}
$$

Then

$$
e^{J_{p} t}=\left[\begin{array}{llll}
e^{J_{p_{1}}\left(\alpha_{1}\right) t} & & & \\
& e^{J_{p_{2}}\left(\alpha_{2}\right) t} & & \\
& & \ddots & \\
& & & e^{J_{p_{v}}\left(\alpha_{v}\right) t}
\end{array}\right] \text { with } p_{1}+p_{2}+\cdots+p_{v}=p
$$

Considering the well-known property $\sqrt{x+y} \leq \sqrt{x}+\sqrt{y}$, it is derived that

$$
\left\|e^{J_{p} t}\right\| \leq\left\|e^{J_{p_{1}}\left(\alpha_{1}\right) t}\right\|+\cdots+\left\|e^{J_{p_{v}}\left(\alpha_{v}\right) t}\right\|=\sum_{i=1}^{v}\left\|e^{J_{p_{i}}\left(\alpha_{i}\right) t}\right\| .
$$

Note that

$$
e^{J_{p_{i}}\left(\alpha_{i}\right) t}=e^{\alpha_{i} t}\left[\begin{array}{ccccc}
1 & t & \frac{t^{2}}{2 !} & \cdots & \frac{t^{\left(p_{i}-1\right)}}{\left(p_{i}-1\right) !} \\
0 & 1 & t & \cdots & \frac{t^{\left(p_{i}-2\right)}}{\left(p_{i}-2\right) !} \\
& & \ddots & \ddots & \vdots \\
& & \ddots & t \\
& & & & \\
& & & & 1
\end{array}\right] .
$$


Consequently, by using some basic properties of the Euclidean norm, we take

$$
\begin{aligned}
\left\|e^{J_{p_{i}}\left(\alpha_{i}\right) t}\right\|= & {\left[\left(e^{\alpha_{i} t}\right)^{2} p_{i}+\left(t e^{\alpha_{i} t}\right)^{2}\left(p_{i}-1\right)+\left(\frac{t^{2}}{2 !} e^{\alpha_{i} t}\right)^{2}\left(p_{i}-2\right)\right.} \\
& \left.+\cdots+\left(p_{i}-\left(p_{i}-1\right)\right)\left(\frac{t^{p_{i}-1}}{\left(p_{i}-1\right) !} e^{\alpha_{i} t}\right)^{2}\right]^{1 / 2} \\
= & {\left[e^{2 \alpha_{i} t} \sum_{m=0}^{p_{i}-1}\left(\left(\frac{t^{m}}{m !}\right)^{2}\left(p_{i}-m\right)\right)\right]^{1 / 2}=e^{\alpha_{i} t}\left[\sum_{m=0}^{p_{i}-1}\left(\left(\frac{t^{m}}{m !}\right)^{2}\left(p_{i}-m\right)\right)\right]^{1 / 2} . }
\end{aligned}
$$

Thus, we conclude to the following result:

$$
\left\|e^{J_{p_{i}}\left(\alpha_{i}\right) t}\right\|=e^{\alpha_{i} t}\left[\sum_{m=0}^{p_{i}-1}\left(\frac{t^{m}}{m !}\right)^{2}\left(p_{i}-m\right)\right]^{1 / 2}
$$

for every $i=1,2, \ldots, v$. Hence, inequality (3.16) holds.

Proposition 3.5. The inequality (3.24) holds

$$
\|\Phi((k-j) T, 0)\| \leq\|Q\| \cdot\left\|Q^{-1}\right\|\left(\left(\sum_{i=1}^{v} e^{\alpha_{i} T}\left(\sum_{m=0}^{p_{i}-1}\left(\frac{T^{m}}{m !}\right)^{2}\left(p_{i}-m\right)\right)^{1 / 2}\right)^{k-j}+\sqrt{q}\right) .
$$

Proof. We known that

$$
\Phi((k-j) T, 0)=Q\left[\begin{array}{cc}
e^{J_{p}(k-j) T} & \mathbb{O}_{p, q} \\
\mathbb{O}_{q, p} & I_{q}
\end{array}\right] Q^{-1}
$$

Then, using also the well-known property of the Euclidean norm $\sqrt{x+y} \leq \sqrt{x}+\sqrt{y}$, we obtain

$$
\begin{aligned}
\|\Phi((k-j) T, 0)\| & \leq\|Q\|\left(\left\|e^{J_{p}(k-j) T}\right\|+\left\|I_{q}\right\|\right)\left\|Q^{-1}\right\| \\
& =\|Q\| \cdot\left\|Q^{-1}\right\|\left(\left\|e^{J_{p}(k-j) T}\right\|+\sqrt{q}\right)
\end{aligned}
$$

since we have $\left\|I_{q}\right\|=\sqrt{\underbrace{1^{2}+1^{2}+\cdots+1^{2}}_{q}}=\sqrt{q}$.

Moreover,

$$
\left\|e^{J_{p}(k-j) T}\right\|=\left\|e^{J_{p} T} e^{J_{p} T} \cdots e^{J_{p} T}\right\| \leq\left(\left\|e^{J_{p} T}\right\|\right)^{k-j}
$$


Considering, now, Lemma 3.4, we have

$$
\left\|e^{J_{p}(\kappa-j) T}\right\| \leq\left(\sum_{i=1}^{v}\left[e^{\alpha_{i} T}\left(\sum_{m=0}^{p_{i}-1}\left(\left(\frac{T^{m}}{m !}\right)^{2}\left(p_{i}-m\right)\right)\right)^{1 / 2}\right]\right)^{k-j} .
$$

Thus, the statement of Proposition 3.5 derives.

Proposition 3.6. The inequality (3.29) holds

$$
\begin{aligned}
\|\Phi(-\lambda, 0)-\Phi(\lambda-T, 0)\| \\
\leq\|Q\| \cdot\left\|Q^{-1}\right\| \\
\cdot\left\{\sum _ { i = 1 } ^ { v } \left[e^{-\alpha_{i} \lambda}\left(\sum_{m=0}^{p_{i}-1}\left(\frac{(-1)^{m} \lambda^{m}}{m !}\right)^{2}\left(p_{i}-m\right)\right)^{1 / 2}\right.\right. \\
\left.\left.+e^{\alpha_{i}(\lambda-T)}\left(\sum_{m=0}^{p_{i}-1}\left(\frac{(\lambda-T)^{m}}{m !}\right)^{2}\left(p_{i}-m\right)\right)^{1 / 2}\right]\right\}
\end{aligned}
$$

Proof. It is known that

$$
\begin{aligned}
\Phi(-\lambda, 0)-\Phi(\lambda-T, 0) & =Q\left[\begin{array}{cc}
e^{-J_{p} \lambda} & \mathbb{O}_{p, q} \\
\mathbb{O}_{q, p} & I_{q}
\end{array}\right] Q^{-1}-Q\left[\begin{array}{cc}
e^{J_{p}(\lambda-T)} & \mathbb{O}_{p, q} \\
\mathbb{O}_{q, p} & I_{q}
\end{array}\right] Q^{-1} \\
& =Q\left[\begin{array}{cc}
e^{-J_{p} \lambda}-e^{J_{p}(\lambda-T)} & \mathbb{O}_{p, q} \\
\mathbb{O}_{q, p} & \mathbb{O}_{p}
\end{array}\right] Q^{-1} .
\end{aligned}
$$

Then, the norm is

$$
\begin{aligned}
\|\Phi(-\lambda, 0)-\Phi(\lambda-T, 0)\| & \leq\|Q\| \cdot\left\|e^{-J_{p} \curlywedge}-e^{J_{p}(\lambda-T)}\right\| \cdot\left\|Q^{-1}\right\| \\
& \leq\|Q\| \cdot\left\|Q^{-1}\right\|\left(\left\|e^{-J_{p} \lambda}\right\|+\left\|e^{J_{p}(\lambda-T)}\right\|\right) .
\end{aligned}
$$

Considering also Lemma 3.4, (3.29) derives.

Now, the whole discussion of this section is completed with the statement of Theorem 3.7 whose proof is based on Lemmas 3.1 and 3.2 and Propositions 3.5 and 3.6. 
Theorem 3.7. The upper bound for the error $\left\|\underline{x}(k T)-\underline{x}_{k}\right\|$, which is derived during the discretization process, is given by

$$
\begin{gathered}
\left\|\underline{x}(k T)-\underline{x}_{k}\right\| \leq \sum_{j=0}^{k-1}\left[\left\{\left(\sum_{i=1}^{v}\left[e^{\alpha_{i} T}\left(\sum_{m=0}^{p_{i}-1}\left(\frac{T^{m}}{m !}\right)^{2}\left(p_{i}-m\right)\right)^{1 / 2}\right]\right)^{k-j}+\sqrt{q}\right\}\right. \\
\cdot \int_{0}^{T}\left\{\sum _ { i = 1 } ^ { v } \left[e^{-\alpha_{i} \lambda}\left(\sum_{m=0}^{p_{i}-1}\left(\frac{(-1)^{m} \cdot \lambda^{m}}{m !}\right)^{2}\left(p_{i}-m\right)\right)^{1 / 2}\right.\right. \\
\left.\left.\left.+e^{\alpha_{i}(\lambda-T)}\left(\sum_{m=0}^{p_{i}-1}\left(\frac{(\lambda-T)^{m}}{m !}\right)^{2}\left(p_{i}-m\right)\right)^{1 / 2}\right]\right\} d \lambda \cdot\left\|\underline{u}_{j}\right\|\right] \\
\cdot\|Q\|^{2} \cdot\left\|Q^{-1}\right\|^{2} \cdot\left\|Q_{n, p}\right\| \cdot\left\|B_{p, l}\right\| .
\end{gathered}
$$

Now, with the upper bound derived by (3.32), we have an analytic expression that penalizes our choice for the sampling period $T$. Although, the above expression is very complicated with a lot of parameters involved, it provides an insightful overview of the error obtained during the discretization (or sampling) process. The following Remark evaluates our upper bound. According to the existing literature, see for instance [14] and references therein, when the sampling period $T$ tends to zero, we are waiting to obtain $\left\|\underline{x}(k T)-\underline{x}_{k}\right\| \rightarrow 0$; that is, the continuous-time solution at time $t=k T$ is exactly the same with the discrete-time points $\underline{x}_{k}$.

Remark 3.8. Consider that the sampling period $T$ tends to zero, that is, $T \rightarrow 0$. In the integral, the function consists exponential and polynomial, continuous functions with respect to $\lambda$. Thus, it is well known from the basic Calculus that

$$
\lim _{x \rightarrow 0} \int_{0}^{x} f(t) d t=\lim _{x \rightarrow 0}(F(x)-F(0))=\lim _{x \rightarrow 0} F(x)-F(0)=F(0)-F(0)=0 .
$$

Moreover, in our case since $T \rightarrow 0$, we obtain

$$
\begin{aligned}
& \lim _{T \rightarrow 0} \int_{0}^{T}\left\{\sum_{i=1}^{\nu}\right. {\left[e^{-\alpha_{i} \lambda}\left(\sum_{m=0}^{p_{i}-1}\left(\frac{(-1)^{m} \cdot \lambda^{m}}{m !}\right)^{2}\left(p_{i}-m\right)\right)^{1 / 2}\right.} \\
&\left.\left.+e^{\alpha_{i}(\lambda-T)}\left(\sum_{m=0}^{p_{i}-1}\left(\frac{(\lambda-T)^{m}}{m !}\right)^{2}\left(p_{i}-m\right)\right)^{1 / 2}\right]\right\} d \lambda=0 .
\end{aligned}
$$


Then

$$
\begin{gathered}
\lim _{T \rightarrow 0} \sum_{j=0}^{k-1}\left[\left\{\left(\sum_{i=1}^{v}\left[e^{\alpha_{i} T}\left(\sum_{m=0}^{p_{i}-1}\left(\frac{T^{m}}{m !}\right)^{2}\left(p_{i}-m\right)\right)^{1 / 2}\right]\right)^{k-j}+\sqrt{q}\right\}\right. \\
\cdot \int_{0}^{T}\left\{\sum _ { i = 1 } ^ { v } \left[e^{-\alpha_{i} \lambda}\left(\sum_{m=0}^{p_{i}-1}\left(\frac{(-1)^{m} \cdot \lambda^{m}}{m !}\right)^{2}\left(p_{i}-m\right)\right)^{1 / 2}\right.\right. \\
\left.\left.\left.+e^{\alpha_{i}(\lambda-T)}\left(\sum_{m=0}^{p_{i}-1}\left(\frac{(\lambda-T)^{m}}{m !}\right)^{2}\left(p_{i}-m\right)\right)^{1 / 2}\right]\right\} d \lambda \cdot\left\|\underline{u}_{j}\right\|\right] \\
\cdot\|Q\|^{2} \cdot\left\|Q^{-1}\right\|^{2} \cdot\left\|Q_{n, p}\right\| \cdot\left\|B_{p, l}\right\|=0
\end{gathered}
$$

since $v, k, p_{i}$ for every $i=1, \ldots, v$ are finite.

Consequently, we have seen that if the sampling period $T$ tends to zero, that is, $T \rightarrow$ 0 , then the solution of the discretized system (1.1), that is, (2.4) (or (2.27)), is an efficient approximation of the relative solution of continuous-time system, that is, (1.13).

\section{Conclusion}

In this paper, we have presented and fully discussed a technique for discretizing efficiently the solution of a linear descriptor (regular) differential input system with consistent initial conditions, and time-invariant coefficients. Additionally, according to the authors' knowledge, for the first time an upper bound for the error $\left\|\underline{x}(k T)-\underline{x}_{k}\right\|$ that derives from the procedure of discretization is provided. This error expression penalizes our choice for the sampling period $T$ through the notion of the $\|\cdot\|$-norm of the difference between the continuous-time solution $\underline{x}(k T)$, at time $t=k T$, and the relevant discrete-time points $\underline{x}_{k}$.

Finally, the results of this paper can be further enriched by the analytic determination of an appropriate sampling period interval, that is, $T \in[a, b]$, which is a future plan of research. An optimal upper bound is also under consideration. Moreover, we are also interested in investigating the case that the non-consistent initial conditions are considered, since distributional solutions are derived.

\section{Acknowledgments}

The project is cofunded by the European Social Fund and National Resources-THALIS I. The authors are very grateful to the anonymous referees for their comments, which improved the quality of the paper. 


\section{References}

[1] K. E. Brenan, S. L. Campbell, and L. R. Petzold, Numerical Solution of Initial-Value Problems in Differential-Algebraic Equations, vol. 14 of Classics in Applied Mathematics, SIAM, Philadelphia, Pa, USA, 1996.

[2] S. L. Campbell, Singular Systems of Differential Equations, vol. 1, Pitman, San Francisco, Calif, USA, 1980.

[3] S. L. Campbell, Singular Systems of Differential Equations, vol. 2, Pitman, San Francisco, Calif, USA, 1982.

[4] L. Dai, Singular Control Systems, vol. 118 of Lecture Notes in Control and Information Sciences, Springer, Berlin, Germany, 1989.

[5] F. L. Lewis, "A survey of linear singular systems," Circuits, Systems, and Signal Processing, vol. 5, no. 1, pp. 3-36, 1986.

[6] V. L. Mehrmann, The Autonomous Linear Quadratic Control Problem: Theory and Numerical Solution, vol. 163 of Lecture Notes in Control and Information Sciences, Springer, Berlin, Germany, 1991.

[7] D. A. Kendrick, "On the Leontief dynamic inverse," Quarterly Journal of Economics, vol. 86, pp. 693696, 1972.

[8] R. G. Kreijger and H. Neudecker, "Kendrick's forward integration method and the dynamic Leontief multisectoral model," Quarterly Journal of Economics, vol. 90, pp. 505-507, 1976.

[9] W. Leontief, "Mathematics in economics," Bulletin of the American Mathematical Society, vol. 60, pp. 215-233, 1954.

[10] W. Leontief, Essays in Economics, M. E. Sharpe, Inc., New York, NY, USA, 1977.

[11] W. Leontief, Input-Output Economics, Oxford University Press, New York, NY, USA, 2nd edition, 1986.

[12] D. A. Livesey, "The singularity problem in the dynamic input-output model," International Journal of Systems Science, vol. 4, pp. 437-440, 1973.

[13] N. P. Karampetakis, "On the discretization of singular systems," IMA Journal of Mathematical Control and Information, vol. 21, no. 2, pp. 223-242, 2004.

[14] G. I. Kalogeropoulos, A. D. Karageorgos, and A. A. Pantelous, "Discretising effectively a linear singular differential system by choosing an appropriate sampling period," IET Control Theory $\mathcal{E}$ Applications, vol. 3, no. 7, pp. 823-833, 2009.

[15] G. Kalogeropoulos, M. Mitrouli, A. Pantelous, and D. Triantafyllou, "The weierstrass canonical form of a regular matrix pencil: numerical issues and computational techniques," in Proceedings of the 4 th International Conference on Numerical Analysis and Its Applications (NAA '09), vol. 5434 of Lecture Notes in Computer Science, pp. 322-329, Springer, Lozenetz, Bulgaria, June 2009.

[16] A. D. Karageorgos, On the study of different qualitative characteristics for generalized control systems, Ph.D. thesis, Athens, Greece, 2009.

[17] R. F. Gantmacher, The Theory of Matrices, Chelsea, New York, NY, USA, 1959.

[18] G. I. Kalogeropoulos, Matrix pencils and linear systems, Ph.D. thesis, City University, London, UK, 1980.

[19] N. Karcanias, "Matrix pencil approach to geometric system theory," Proceedings of the IEE, vol. 126, no. 6, pp. 585-590, 1979.

[20] N. Karcanias and G. E. Hayton, "Generalised autonomous differential sys-tems, algebraic duality, and geometric theory," in Proceedings of the 8th IFAC Triennial World Congress, Kyoto, Japan, 1981.

[21] A. Rachid, "A remark on the discretization of singular systems," Automatica, vol. 31, no. 2, pp. 347348, 1995.

[22] P. Van Dooren, Reducing Subspaces: Definitions, Properties, and Algorithms, in Matrix Pencils, vol. 973 of Lecture Notes in Mathematics, Springer, Berlin, Germany, 1983.

[23] E. Grispos, Study of generalized linear differential systems with the use of matrix Pencil theory, Ph.D. thesis, Department of Mathematics, Athens University, Athens, Greece, 1991.

[24] F. N. Koumboulis and B. G. Mertzios, "On Kalman's controllability and observability criteria for singular systems," Circuits, Systems, and Signal Processing, vol. 18, no. 3, pp. 269-290, 1999.

[25] D. G. Luenberger and A. Arbel, "Singular dynamic Leontief systems," Econometrica, vol. 45, pp. 991995, 1977. 


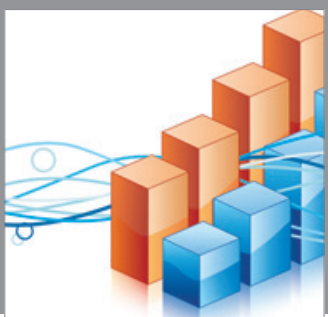

Advances in

Operations Research

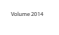

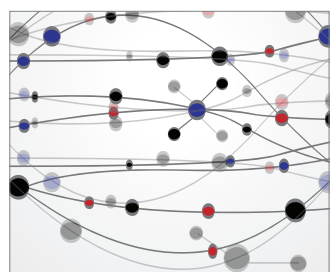

\section{The Scientific} World Journal
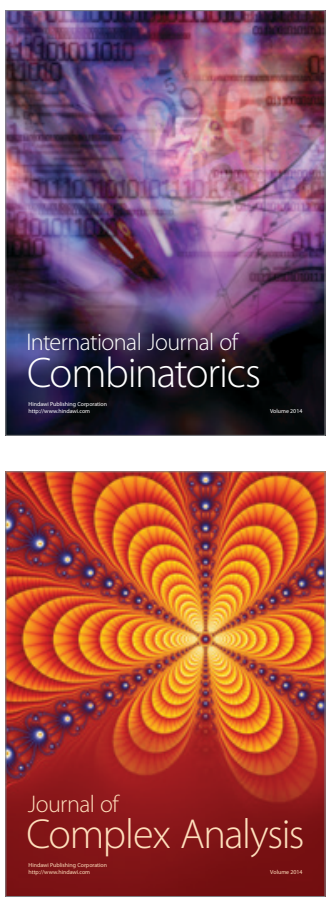

International Journal of

Mathematics and

Mathematical

Sciences
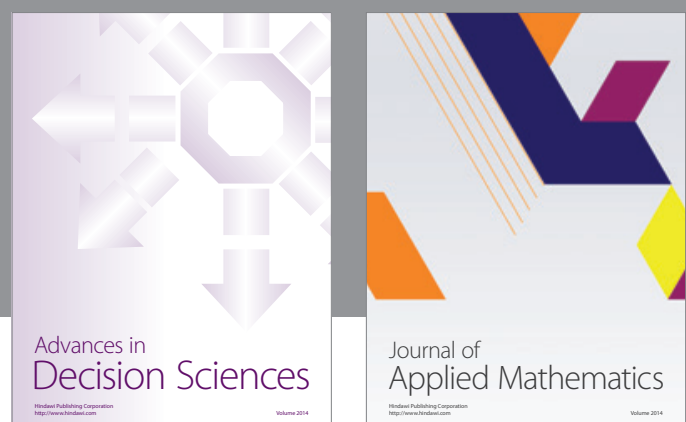

Journal of

Applied Mathematics
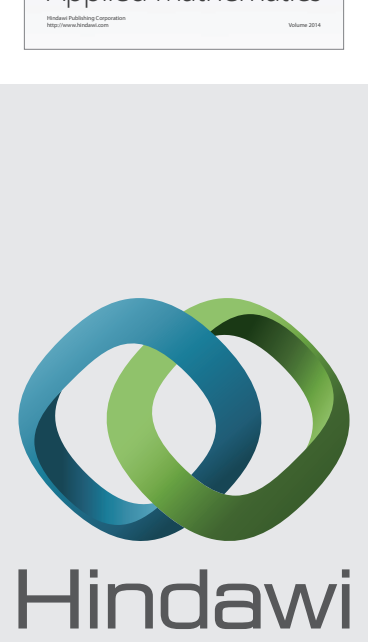

Submit your manuscripts at http://www.hindawi.com
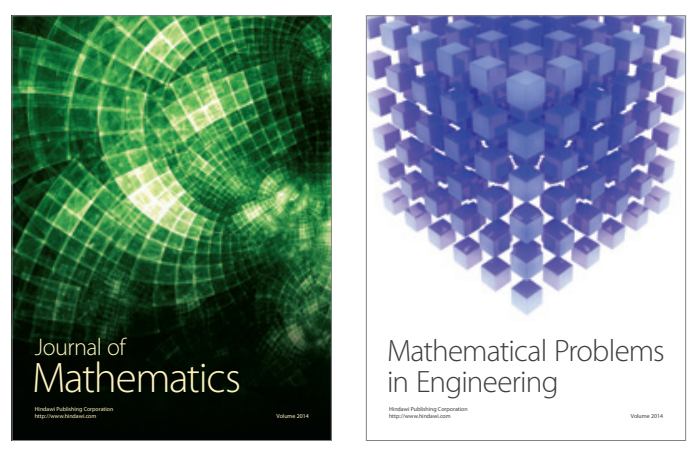

Mathematical Problems in Engineering
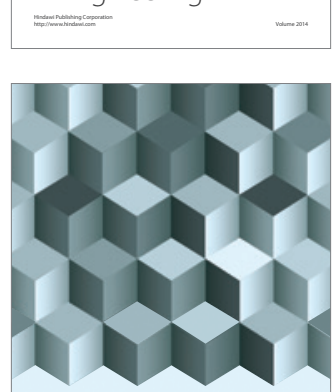

Journal of

Function Spaces
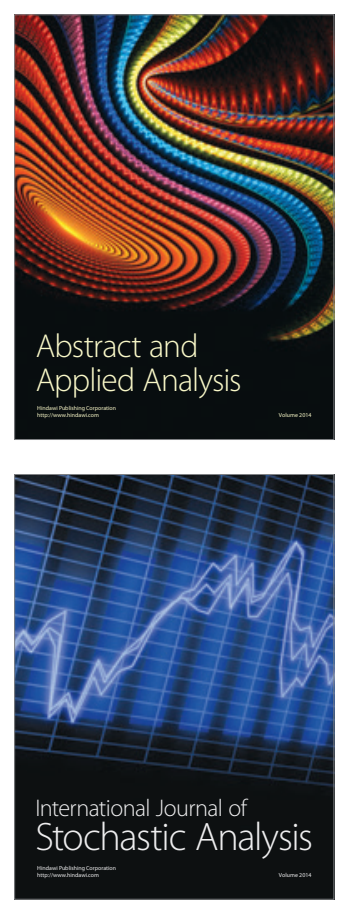

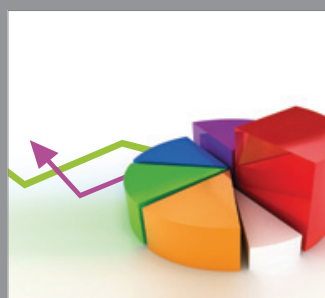

ournal of

Probability and Statistics

Promensencen
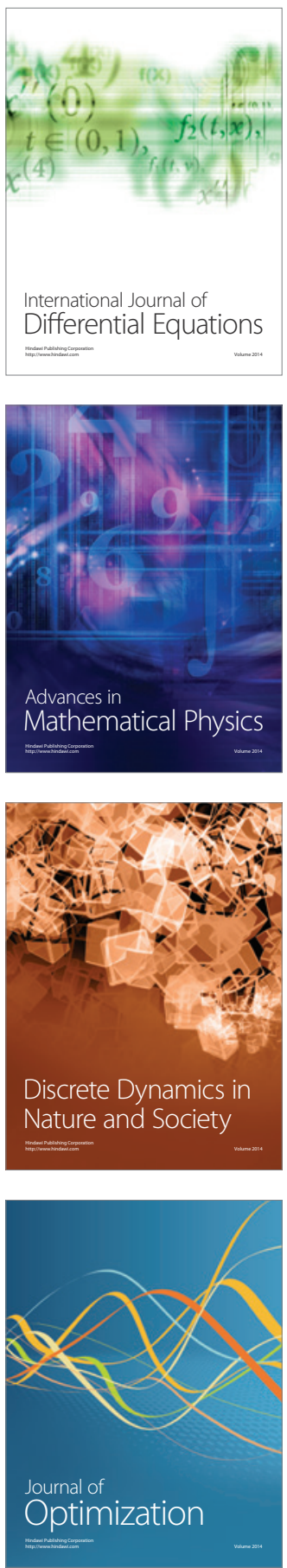